\section{Radial basis function network assisted space-time equalisation for dispersive fading environments}

A. Wolfgang, S. Chen and L. Hanzo

A novel radial basis function network assisted decision-feedback aided space-time equaliser designed for receivers employing multiple antennas is presented. The proposed receiver structure outperforms the linear minimum mean-squared error benchmarker and is less sensitive to both error propagation and channel estimation errors.

Introduction: The capability of receivers employing multiple antennas to increase the achievable system capacity and to suppress the effects of co-channel interference has motivated intense research in the field of space-time equalisation [1]. Most contributions, however, focus on sub-optimal linear receivers or investigate the performance of maximum-likelihood sequence estimators (MLSE), which suffer from an exponentially increasing complexity against the delay-spread encountered. Because it encounters non-minimum phase channels, the received signal constellation may become linearly non-separable and to counteract this problem we introduce a novel nonlinear radial basis function network (RBFN) [2] assisted space-time equaliser (STE) for uplink communication scenarios. For the sake of complexity reduction an RBF-aided decision feedback (DF) structure is used, which necessitates the detection of all users. The investigated scenario assumes multiple users communicating with the basestation (BS) over singleinput multiple-output (SIMO) Rayleigh fading channels.

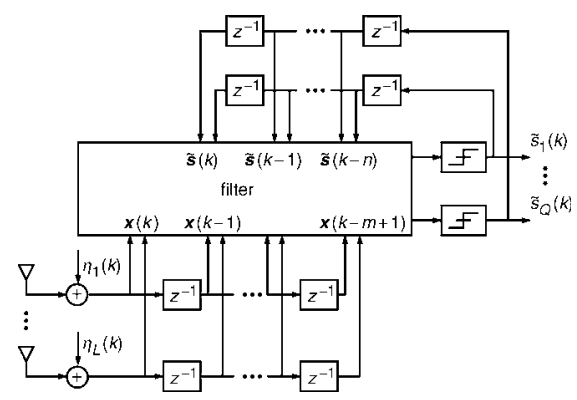

Fig. 1 General structure of decision feedback aided space-time equaliser employing $L$ receive antennas with feedforward order $m$ and feedback order $n$ The signals $s_{q}(k)$ of all $Q$ users are detected

System model: The system considered consists of $Q$ binary phase shift keying (BPSK) modulated sources and a BS receiver, which is assumed to employ $L$ antennas. The channel output signal of the $l$ th antenna element at time instant $k$ can then be written as:

$$
x_{l}(k)=\sum_{q=1}^{Q} \sum_{n=0}^{K} h_{l q, n} s_{q}(k-n)+\eta(k)
$$

where $h_{l q, n}$ is the complex valued channel gain of the $n$th multipath component describing the channel between the $q$ th source and the $l$ th receiver antenna, $K$ is the number of multipath components and $\eta(k)$ is the complex valued additive white Gaussian noise (AWGN) having a variance of $\varepsilon\left[\left|\eta_{l}(k)\right|^{2}\right]=2 \sigma^{2}$. Each of the receiver's antenna elements is followed by a tapped delay line of length $m$, which is also referred to as the feedforward section of the RBF-aided STE, as shown in Fig. 1. In vectorial notation, the channel output can be expressed by the supervector $\boldsymbol{x}(k)=\left[\boldsymbol{x}(k)^{T}, \ldots, \boldsymbol{x}(k-m+1)^{T}\right]^{T}$, where $\boldsymbol{x}(k)$ is a column vector hosting the $L$ number of antenna-element output signals $x_{l}(k)$ given in (1) (please note the different fonts in the notation). The relation between the signal transmitted by the $Q$ sources and the channel output for channel tap $n$ is described by a $(L \times Q)$-dimensional matrix $\boldsymbol{H}_{n}$ where the $(l q)$ th element of the matrix is given as $h_{l q, n}$. The super-matrix $\boldsymbol{H}$ representing the total system can then be obtained by concatenating the $(L \times Q)$-dimensional matrices $\boldsymbol{H}_{n}$, yielding:

$$
\boldsymbol{H}=\left(\begin{array}{cccccc}
\boldsymbol{H}_{n} & \cdots & \boldsymbol{H}_{n-m+1} & 0 & \cdots & 0 \\
& & \ddots & & \ddots & \\
0 & \cdots & 0 & \boldsymbol{H}_{n} & \cdots & \boldsymbol{H}_{n-m+1}
\end{array}\right)
$$

The channel output vector $\boldsymbol{x}(k)$ can now be expressed as

$$
\begin{aligned}
\boldsymbol{x}(k) & =\boldsymbol{H}\left[\boldsymbol{s}(k)^{T}, \ldots, \boldsymbol{s}(k-m+1)^{T}\right]^{T}+\left[\eta_{1}(k)^{T}, \ldots, \eta_{L}(k)^{T}\right]^{T} \\
& =\boldsymbol{H} \boldsymbol{s}(k)+\eta(k) \\
& =\overline{\boldsymbol{x}}(k)+\eta(k)
\end{aligned}
$$

where $\boldsymbol{s}(k)=\left[s_{1}(k), \ldots, s_{Q}(k)\right]^{T}$ is a column vector containing the symbols transmitted by the $Q$ sources and $\eta_{l}(k)=\left[\eta_{1}(k), \ldots, \eta_{l}(k-\right.$ $m+1)]^{T}$. Assuming that all sources transmit with identical power, the signal-to-noise ratio (SNR) of user $q$ is given as $\mathrm{SNR}_{q}=$ $\left(\sum_{l=1}^{L} \sum_{n=0}^{K} \varepsilon\left[\left|h_{l q, n}\right|^{2}\right] / 2 \sigma^{2}\right)$.

DF-STE: The performance of both linear and nonlinear equalisers can be enhanced by incorporating a decision feedback structure in the receiver [2], as shown in Fig. 1. In addition to the feedforward section, the DF-STE is then characterised by the decision delay $\tau$ and the decision feedback order $n$. Note that the oldest symbol vector, which still influences the detected symbol $\tilde{s}_{q}(k-\tau)$ is $s(k-m+1-K)$. Furthermore, the oldest feedback symbol vector is $s(k-\tau-n)$. Without loss of generality we therefore chose $n=m+K-1-\tau$ for the derivation of the proposed DF-STE. In order to describe the feedback structure, we first divide the system matrix $\boldsymbol{H}$ into two sub-matrices $\boldsymbol{H}=\left[\begin{array}{ll}\boldsymbol{H}_{1} & \boldsymbol{H}_{2}\end{array}\right]$, where $\boldsymbol{H}_{1}$ hosts the first $Q(\tau+1)$ columns of $\boldsymbol{H}$ and $\boldsymbol{H}_{2}$ represents the last $Q_{n}$ columns in $\boldsymbol{H}$. The array output can then be written as:

$$
\boldsymbol{x}(k)=\boldsymbol{H}_{1} \boldsymbol{s}_{1}(k)+\boldsymbol{H}_{2} \boldsymbol{s}_{2}(k)+\eta(k)
$$

where $\boldsymbol{s}_{1}(k)=\left[\boldsymbol{s}(k)^{T}, \ldots, \boldsymbol{s}(k-\tau)^{T}\right]^{T}$ indicates the symbols in the feedforward shift register and $\boldsymbol{s}_{2}(k)=\left[s(k-\tau-1)^{T}, \ldots, s(k-\tau-n)^{T}\right]^{T}$ denotes the symbols in the feedback register. Under the assumption that the feedback vector is correct, (3) can be rewritten as:

$$
r(k)=\boldsymbol{x}(k)-\boldsymbol{H}_{2} \tilde{\mathbf{s}}_{2}(k)=\boldsymbol{H}_{1} \boldsymbol{s}_{1}(k)+\eta(k)
$$

where $\boldsymbol{r}(k)$ is the observation space owing to the decision feedback. For a given feedback vector the possible noise-free channel output states in this new observation space $\overline{\boldsymbol{r}}(k)$ may assume $n_{s}=2^{Q(\tau+1)}$ different values, depending on the transmitted symbol vector $\boldsymbol{s}^{(i)}, 1 \leq i \leq n_{s}$, yielding $\overline{\boldsymbol{r}}^{(i)}=\boldsymbol{H}_{1} \boldsymbol{s}_{1}^{(i)}$. The set of all possible desired output states in the translated space $\overline{\boldsymbol{r}}(k)$ can be partitioned into two subsets $\mathcal{R}_{q}^{ \pm}$, depending on the binary value of the transmitted symbol $s_{q}^{(i)}(k-\tau)$ of the desired user $q$ as:

$$
\mathcal{R}_{q}^{ \pm}=\left\{\bar{r}_{q}^{(i, \pm)}=\boldsymbol{H}_{1} \boldsymbol{s}_{1}^{(i)} \text { if } s_{q}^{(i)}(k-\tau)= \pm 1\right\}
$$

Based on the space translation given in (4), the decision function of the filter depicted in Fig. 1 can be written as:

$$
\tilde{s}_{q}(k-\tau)=\operatorname{sgn}\left(f_{B, q}(\boldsymbol{r})\right)= \begin{cases}+1 & \text { if } f_{B, q}(\boldsymbol{r}(k)) \geq 0 \\ -1 & \text { if } f_{B, q}(\boldsymbol{r}(k))<0\end{cases}
$$

where the optimal Bayesian decision function [2] $f_{B, q}(\cdot)$ based on the difference of the associated conditional density functions is given as:

$$
\begin{aligned}
f_{B, q}(\boldsymbol{r}(k))= & P\left(\boldsymbol{x}(k) \mid s_{q}(k-\tau)=+1\right)-P\left(\boldsymbol{x}(k) \mid s_{q}(k-\tau)=-1\right) \\
= & \sum_{\overline{\boldsymbol{r}}_{q}^{(i,)} \in \mathcal{R}_{q}^{+}} p^{(i,+)} p\left(\boldsymbol{r}(k)-\overline{\boldsymbol{r}}_{q}^{(i,+)}\right)-\sum_{\overline{\boldsymbol{r}}_{q}^{(i,-)} \in \mathcal{R}_{q}^{-}} p^{(i,-)} p\left(\boldsymbol{r}(k)-\overline{\boldsymbol{r}}_{q}^{(i,-)}\right) \\
= & \sum_{\overline{\boldsymbol{r}}_{q}^{(i,+)} \in \mathcal{R}_{q}^{+}} p^{(i,+)} \frac{1}{\left(2 \pi \sigma^{2}\right)^{-L m}} \exp \left(-\frac{\left\|\boldsymbol{r}(k)-\overline{\boldsymbol{r}}_{q}^{(i,+)}\right\|^{2}}{2 \sigma^{2}}\right) \\
& -\sum_{\overline{\boldsymbol{r}}_{q}^{(i,-)} \in \mathcal{R}_{q}^{-}} p^{(i,-)} \frac{1}{\left(2 \pi \sigma^{2}\right)^{-L m}} \exp \left(-\frac{\left\|\boldsymbol{r}(k)-\overline{\boldsymbol{r}}_{q}^{(i,-)}\right\|^{2}}{2 \sigma^{2}}\right)
\end{aligned}
$$

where $\overline{\boldsymbol{x}}_{i}^{q, \pm} \in \mathcal{R}^{q, \pm}, p^{(i,+)}$ and $p^{(i,-)}$ are the a priori probabilities of $\boldsymbol{r}_{i}^{(q,+)}$ and $\boldsymbol{r}_{i}^{(q,-)}$, respectively, while $p(\cdot)$ is the PDF of $\boldsymbol{\eta}(k)$. The Bayesian DFSTE can be realised using a RBFN employing a Gaussian kernel. The response of such a RBFN is given as:

$$
\begin{aligned}
f_{R B F}(\boldsymbol{r}(k)) & =\sum_{i=1}^{N_{c}} w_{i} \phi\left(\boldsymbol{r}(k), \boldsymbol{c}^{(i)}\right) \quad \text { with } \quad \phi\left(\boldsymbol{r}(k), \boldsymbol{c}^{(i)}\right) \\
& =\exp \left(-\frac{\left\|\boldsymbol{r}(k)-\boldsymbol{c}^{(i)}\right\|^{2}}{\rho}\right)
\end{aligned}
$$


where the RBF centres $c^{(i)}$ are set to the possible noise-free channel output states determined by the channel impulse response (CIR), the radius $\rho$ is chosen to be $2 \sigma^{2}$ and the weights $w_{i}$ are set to +1 , if we have $c(i) \in \mathcal{R}^{q,+}$ and to -1 if $c^{(i)} \in \mathcal{R}^{q,-}$. For the detection of $\tilde{s}_{q}(k-\tau)$ the received signal vector $\boldsymbol{x}(k)$ is transformed into the translated space $\boldsymbol{r}(k)$ by subtracting the product of the feedback sequence $\tilde{\boldsymbol{s}}_{2}(k)$ and $\boldsymbol{H}_{2}$, given in (4). In the translated space the signal is detected using the RBFN given in (8).

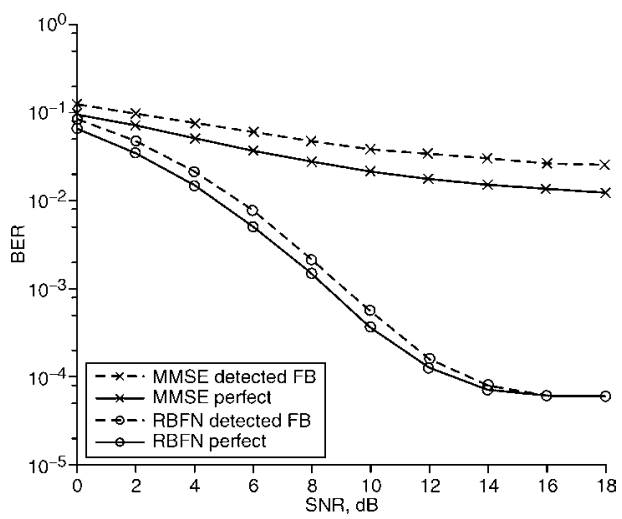

Fig. 2 Average BER of three users against SNR for detected and correct feedback

The receiver was assumed to have perfect channel knowledge. A normalised Doppler frequency of $f_{d}=0.0005$ and identical CIRs given as

$h_{l q}=\sqrt{ }(0.5)+\sqrt{ }(0.5) z^{-1}$ were used. All three users transmitted at an equal power. The receiver parameters were chosen to be $L=2, m=2$ and $\tau=1$

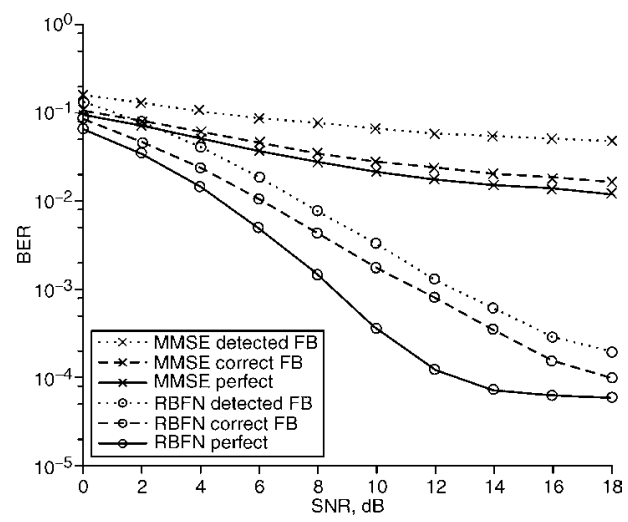

Fig. 3 Average BER of three users against SNR for detected and correct feedback

The receiver used estimated channel state information. A normalised Doppler frequency of $f_{d}=0.0005$ and identical CIRs given as $h_{l q}=\sqrt{ }(0.5)+\sqrt{ }(0.5) z^{-1}$ were used. All three users transmitted at an equal power. The receiver parameters were chosen to be $L=2, m=2$ and $\tau=1$. The label 'perfect' indicates correct feedback and perfect CIR knowledge
Results: The system considered in our study consists of three equalpower users having identical unfaded CIRs of $h_{l q}(z)=\sqrt{ }(0.5)+$ $\sqrt{ }(0.5) z^{-1}$. Each CIR tap was subjected to independent Rayleigh fading having an identical normalised Doppler frequency of $f_{d}=$ 0.0005 for all users. In our study we consider a transmission frame consisting of 20 training and 180 payload symbols. The channel estimator uses the training symbols for estimating the channel matrix $\boldsymbol{H}(k)$, which is then passed to the STE. A Kalman filter is employed for channel estimation, which predicts the CIR coefficients needed in the feedforward (FF) section and estimates the channel for the feedback (FB) section, as proposed in [3]. After the training period the channel estimator switches to decision directed mode to track the channel. It is important to mention that, in order to be able to perform decision feedback equalisation, all users' signals have to be detected. The performance of the RBFN assisted DFSTE is compared to that of the linear MMSE DF-STE of [1].

Fig. 2 shows the average BER of all users for perfect channel estimation. We can see that the nonlinear RBFN assisted DF-STE outperforms the linear MMSE. It is also seen that the nonlinear receiver suffers very little from FB-induced error-propagation. The residual BER of the MMSE receiver indicates a high number of linearly nonseparable signal constellation points at the channel's output induced by the fading. The non-separable constellation points for the RBFN aided receiver are significantly less frequent, as indicated by the lower residual BER. The effects of estimated, rather than perfect CIRs, are illustrated in Fig. 3, where it can be observed that the RBFN receiver is also more robust against channel estimation errors.

Conclusions: The results demonstrate that the RBFN assisted DFSTE outperforms the linear MMSE benchmarker and its performance is less degraded by both error propagation and CIR estimation errors. Its performance is mainly limited by the tracking ability of the Kalman CIR estimator.

(C) IEE 2004

10 May 2004

Electronics Letters online no: 20045374

doi: 10.1049/el:20045374

A. Wolfgang, S. Chen and L. Hanzo (School of ECS, University of Southampton, Southampton SO17 1BJ, United Kingdom)

\section{References}

1 Tidestav, C., Sternad, M., and Ahlen, A.: 'Reuse within a cell-interference rejection or multiuser detection?', IEEE Trans. Commun., 1999, 47, (10), pp. $1511-1522$

2 Hanzo, L., Wong, C.H., and Yee, M.S.: 'Adaptive wireless transceivers: turbo-coded, turbo-equalized and space-time coded TDMA, CDMA, and OFDM systems' (John Wiley and IEEE Press, 2002)

3 Komninakis, C., et al.: 'Multi-input multi-output fading channel tracking and equalization using Kalman estimation', IEEE Trans. Signal Process., 2002, 50, (5), pp. 1065-1076 